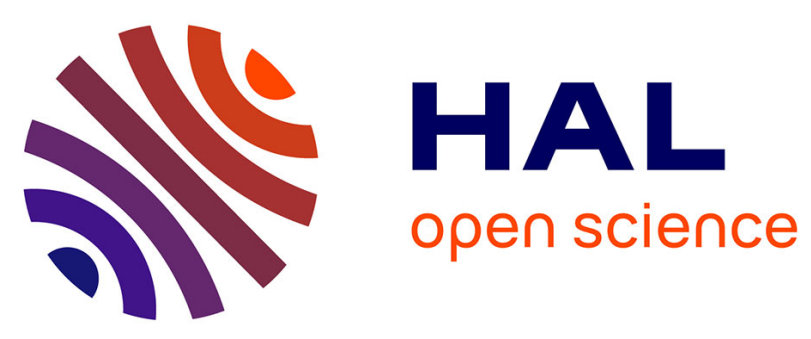

\title{
La précaution réglementaire
}

Henri Boullier, Brice Laurent

\section{To cite this version:}

Henri Boullier, Brice Laurent. La précaution réglementaire: Un mode européen de gouvernement des objets techniques. Politique européenne, 2015, 49 (49), pp.30. 10.3917/poeu.049.0030 . halshs$01242949 \mathrm{v} 2$

\section{HAL Id: halshs-01242949 \\ https://shs.hal.science/halshs-01242949v2}

Submitted on 22 May 2019

HAL is a multi-disciplinary open access archive for the deposit and dissemination of scientific research documents, whether they are published or not. The documents may come from teaching and research institutions in France or abroad, or from public or private research centers.
L'archive ouverte pluridisciplinaire HAL, est destinée au dépôt et à la diffusion de documents scientifiques de niveau recherche, publiés ou non, émanant des établissements d'enseignement et de recherche français ou étrangers, des laboratoires publics ou privés. 


\section{POLITIQUE EUROPÉENNE \\ $N^{\circ} 49$ | 2015}

Henri Boullier et Brice Laurent

[p. 30-53]

\section{La précaution réglementaire. Un mode européen de gouvernement des objets techniques}

Cet article s'intéresse au fonctionnement concret du règlement européen REACH sur les substances chimiques. En analysant les opérations construisant des catégories réglementaires définissant des objets techniques et organisant la répartition des pouvoirs entre acteurs publics et privés, européens et nationaux, ce texte suggère que la notion de « précaution réglementaire » permet de caractériser un mode de gouvernement européen des substances chimiques. Le cas de la procédure d'enregistrement des nanomatériaux et celui de l'autorisation des phtalates mettent en évidence le réglage entrepris par la Commission pour mettre en place des approches «au cas par cas », multipliant les catégories réglementaires au prix d'une réticence à introduire des contraintes légales. Ces initiatives ne sont pas consensuelles. Des États membres et le Parlement européen construisent des catégories réglementaires plus rigides, et par là proposent un mode alternatif de gouvernement des substances chimiques. Au final, la précaution réglementaire permet de décrire les effets constitutionnels des catégories réglementaires pour les objets techniques européens.

\section{Regulatory precaution. A European mode of governing technical objects}

This paper focuses on the European REACH regulation on chemicals. We analyze the operations crafting regulatory categories in order to define technical objects and organizing the distribution of powers between public and private, national and European actors. This article suggests that the concept of "regulatory precaution" helps characterize a European mode of governing chemicals. The examples of the registration of nanomaterials and that of the autorisation of phtalates highlight the choice made by the European Commission in favour of a "case by case approach" that ends up multiplying regulatory categories, while postponing the introduction of legal constraints. These initiatives are not consensual: member states and the European Parliament produce more rigid regulatory categories, and thereby suggest an alternative mode of governing chemicals. Thus, regulatory precaution offers a lens through which one can identify the constitutional effects of the regulatory categories for European technical objects. 


\title{
La précaution réglementaire \\ Un mode européen de gouvernement des objets techniques ${ }^{1}$
}

\author{
Henri Boullier \\ Université Paris-Est \\ Brice Laurent \\ Mines Paris Tech
}

a «précaution » est devenue une exigence forte de l'action publique européenne. Le principe de précaution, mentionné dès le traité de Maastricht en 1992, fait émerger des discussions relatives à l'évaluation du risque, aux modalités de la gestion de l'incertitude scientifique, et contribue à redéfinir les rapports entre science et politique dans les institutions européennes (Commission européenne, 2000 ; Dratwa, 2002 ; Levidow, 2001). Pour certains commentateurs, l'usage de la précaution au sein des institutions européennes est « arbitraire et capricieux » (Marchant et Mossman, 2004). Pour d'autres, l'objectif de précaution est le signal d'une évolution des institutions européennes, devenues plus sensibles au risque que l'État fédéral américain (Vogel, 2012). Le débat porte sur la nature et les effets des dispositifs réglementaires potentiellement contraignants pour des activités industrielles caractérisées par des risques à mesurer et contrôler. Il fait écho à une littérature plus large sur le principe de précaution, marquée elle aussi par une opposition entre des critiques (déplorant les « blocages » induits par la précaution ${ }^{2}$ ou à l'inverse l'incapacité à prendre en compte les catastrophes (Dupuy, 2003 ; cf. Larrère, 2003 sur cette opposition) et les défenseurs d'une version «proportionnée » du principe de précaution, favorable à l'action,

Ce texte est issu de travaux préliminaires présentés au cours des conférences conjointes de l'European Association for the Study of Science and Technology et de la Society for Social Studies of Science qui se sont déroulées à Copenhague (17-20 octobre 2012). Les auteurs remercient par ailleurs pour leurs relectures et leurs remarques Benjamin Lemoine et Stève Bernardin, ainsi que les évaluateurs de la revue pour leurs commentaires.

2 Pour une critique du « mésusage » du principe de précaution voir (Loubière et al., 2001). 
notamment en matière de recherche (Godard, 1999, 2003). Au sein des institutions européennes, ces discussions sont à mettre en regard de la volonté d'optimisation de la contrainte réglementaire européenne, qui se traduit par un objectif dit de «meilleure réglementation » (better regulation) visant à limiter la production normative.

Ce texte introduit et utilise l'expression «précaution réglementaire » pour désigner à la fois l'opérationnalisation d'un objectif de précaution par des instruments réglementaires européens (notamment dans le cadre de politiques sanitaires ou environnementales), et l'usage précautionneux de la contrainte réglementaire. Notre objectif n'est pas de rendre compte de façon exhaustive des multiples références au «principe de précaution » dans les textes réglementaires européens (de Sadeleer, 2001; Tosun, 2013) ou dans les procès (Stokes, 2008). Il consiste plutôt, à la suite de certains travaux ayant souligné les redéfinitions des opérations de gouvernement dans le cadre d'un objectif de précaution (Lascoumes, 1996), à mettre en évidence un mode européen de gouvernement des objets techniques s'appuyant sur la précaution. La précaution réglementaire permet de rendre compte d'une double injonction à l'anticipation des risques en situation d'incertitude et à la limitation de la contrainte règlementaire. À cet égard, elle permet de mettre en évidence les processus qui sont censés permettre d'atteindre l'objectif général de précaution présent dans les textes européens, et qui ce faisant participent à le définir comme une modalité d'action étroitement articulée à l'évaluation et à la gestion des risques ( $c f$. Stokes, 2008).

L'enquête que nous présentons ${ }^{3}$ ici s'appuie sur l'analyse du règlement REACH (règlement sur l'Enregistrement, l'Evaluation, l'Autorisation et les Restrictions des substances chimiques), qui organise le contrôle du marché des substances chimiques en Europe dans un objectif explicite de mise en œuvre du principe de précaution. Le règlement est unique par la taille du marché concerné (celui des substances chimiques) et l'ampleur de l'initiative, « la plus complexe dans l'histoire de l’Union " $^{4}$, qui a donné lieu à la création d'enquête conduit entre 2010 et 2013 auprès d'acteurs impliqués dans la mise en œuvre de REACH. Nous mobilisons ici des matériaux collectés au cours d'entretiens auxquels s'est ajoutée une analyse de documents produits et utilisés par les experts, fonctionnaires nationaux, eurofonctionnaires, parlementaires, industriels et lobbyistes que nous avons interviewés. 
de l'Agence européenne des produits chimiques (ECHA), et a profondément redéfini les obligations des industriels, responsables à présent de démontrer que les risques des substances qu'ils produisent ou commercialisent sont traités de façon adéquate (Fischer, 2008; Jouzel et Lascoumes, 2011). $\mathrm{REACH}$ est une illustration particulièrement parlante pour rendre compte d'un ensemble d'initiatives des institutions européennes marqué à la fois par les exigences de précaution et de compétitivité économique, de contrôle réglementaire et de «meilleure réglementation » (Fuchs, 2009).

Dans le cadre de REACH, l'intervention des institutions européennes s'exerce au niveau de la construction de catégories réglementaires permettant de faire des substances chimiques des objets gouvernables. Les catégories réglementaires sont autant de dispositifs qui permettent d'instrumenter le règlement $\mathrm{REACH}$. La production de catégories est en effet nécessaire pour rendre gouvernables les substances chimiques : il faut pouvoir repérer les substances et les identifier grâce à des catégories stables afin de mettre en place des dispositifs d'étiquetage ou de contrôle (Lezaun, 2006). L'exercice est difficile, car il impose aux acteurs de se repérer parmi la multitude de substances produites par l'industrie chimique et de s'accorder sur des contraintes juridiques. REACH nous permettra ainsi de lier l'étude de l'organisation politique européenne et celle de la fabrication d'objets gouvernables au sein de cette organisation (Bowker et Star, 1999; Jasanoff, 2004). Nous verrons que la précaution réglementaire, en multipliant les cas particuliers et les lieux de la discussion entre parties prenantes, est une opération qui articule étroitement la manufacture d'objets gouvernables avec la mise en ordre politique de l'espace européen.

Les cas des nanomatériaux et des phtalates permettent d'illustrer successivement le fonctionnement de deux des procédures introduites dans REACH - l'« enregistrement » et l'« autorisation ». Un industriel distribuant ou produisant une substance en Europe doit l'« enregistrer » grâce à un dossier envoyé à l'ECHA. Les nanomatériaux constituent un domaine où l'enregistrement se révèle particulièrement délicat. Leur point commun est la présence de nouvelles propriétés physico-chimiques liées à la faible taille de leurs composantes. Ces propriétés leur confèrent un intérêt pour des applications industrielles, mais suscitent également des interrogations relatives à d'éventuels risques sanitaires. Dans le cadre de la mise en œuvre de REACH, la définition de la catégorie «nanomatériaux » est controversée: faut-il ou non créer une nouvelle catégorie réglementaire qui permettrait d'embrasser l'ensemble des nanomatériaux, et dans ce cas, comment la 
définir? L'exemple des phtalates nous permet d'illustrer les cas, bien moins nombreux ${ }^{5}$, de substances particulièrement préoccupantes soumises à la procédure dite « d'autorisation ${ }^{6}$. Contrairement à ce que son nom laisse entendre, il s'agit d'une procédure qui interdit a priori tous les usages d'une substance, sauf autorisation accordée au cas par cas. « Autoriser » les phtalates au sens de REACH revient à démontrer qu'un ensemble bien connu de substances dangereuses peut être éliminé du marché européen - au prix, on le verra, d’ajustement des catégories réglementaires.

Ces exemples nous permettront de mettre en évidence un mode de gouvernement, la « précaution réglementaire », qui consiste à multiplier les cas particuliers, sur la définition des substances et de leurs usages, de telle sorte que l'intervention publique développe une position hybride, qui n'est réductible ni à la solution de la pure contrainte réglementaire ni à la délégation de l'organisation du marché des substances chimiques à des acteurs privés. Ce réglage des catégories réglementaires, permettant de faire fonctionner les procédures d'enregistrement et d'autorisation en limitant les contraintes sur les industriels européens, n'est pas pour autant consensuel. Des initiatives émanant des États membres et du Parlement mettent en évidence des possibilités de construction de catégories réglementaires différentes de celles prévues par REACH. En proposant des modes d'action publique au sein desquels la précaution donne lieu à la construction de catégories rigides et à la contrainte réglementaire volontaire, ces initiatives suggèrent des voies alternatives à la précaution réglementaire.

\section{Enregistrer les nanomatériaux}

\section{L’approche « au cas par cas »}

En 2009, une Communication de la Commission européenne explique qu'aucune nouvelle catégorie d'objets « nano » ne doit être créée dans le droit européen, mais qu'il suffit d'adapter la réglementation existante relative aux substances chimiques aux particularités des nanomatériaux. Elle propose d'utiliser REACH en adoptant une démarche « au cas pas cas », dont

5 Environ 1500 substances sont concernées par la procédure d'autorisation quand plus de 30000 entrent dans le champ de l'enregistrement. 
la logique est que le traitement des nanomatériaux n'a aucune raison de différer de l'enregistrement de n'importe quelle autre substance. La logique de cette démarche consiste à utiliser la réglementation dans un objectif de précaution tout en manipulant avec précaution la contrainte réglementaire. Cette « précaution réglementaire » se manifeste par la réticence à modifier REACH et par l'objectif d'adaptation de la contrainte à chaque « cas » d'enregistrement d'un nanomatériau.

Pour comprendre les implications de la précaution réglementaire ici, il est nécessaire d'examiner la procédure d'enregistrement. Celle-ci consiste à soumettre un dossier à l'ECHA dans lequel l'entreprise productrice ou utilisatrice démontre en faisant référence à des études scientifiques qu'elle maîtrise les risques de la substance à enregistrer. Certaines substances voient l'exigence d'enregistrement décalée dans le temps, selon un calendrier qui varie suivant les quantités concernées. Ces « substances bénéficiant d’un régime transitoire » sont celles qui sont déjà fabriquées ou sur le marché européen au $1^{\mathrm{er}}$ mai 2004, ou figurant à l'inventaire des substances chimiques existant sur le marché communautaire. Pour les substances ne bénéficiant pas du régime transitoire, l'industriel doit fournir un dossier technique comprenant des informations sur l'identité de la substance, sa fabrication et son utilisation, ainsi qu'un rapport sur la sécurité chimique. L’approche «au cas par cas » proposée par la Commission pour les nanomatériaux consiste donc à s'interroger, pour chaque nanomatériau, s’il peut être identifié à une substance existante ou s'il doit faire l'objet d'un enregistrement spécifique.

Le cas des nanomatériaux est révélateur à cet égard, car il entraîne de nombreuses difficultés pratiques. Ces difficultés ont été soulevées par les critiques de l'approche « au cas par cas », émanant, notamment, du Bureau européen de l'environnement (BEE), qui fédère les organisations environnementales européennes pour intervenir dans la réglementation européenne. Elles ont également été visibles dans les travaux de l'ECHA consacrés à la mise en œuvre de REACH pour les nanomatériaux. Ces critiques soulignent deux types de problèmes ${ }^{7}$. 
Une première difficulté apparaît clairement: comment différencier la version « nano » d'une particule de sa version « non nano »? Le dioxyde de titane par exemple existe sous des formes « nano » et sous des formes « non nano ». Les deux formes diffèrent par la taille et ont des propriétés différentes. En conséquence, et c'est la question que se posent les services de l'Agence européenne des substances chimiques, les risques qu'elles entraînent pour la santé humaine ou l'environnement pourraient être différents ${ }^{8}$. Mais la taille n'est pas un critère utilisé dans REACH pour différencier entre des substances de même formule chimique. Comment alors, dans le cadre de la réglementation, distinguer entre un dioxyde de titane " nano » et un dioxyde de titane « non nano »? Deuxième difficulté, comment différencier deux nanomatériaux? La distinction peut être effectuée si leur composition chimique est différente. Comment faire lorsqu'elle est similaire et que les objets diffèrent seulement par leur forme? Par exemple, les nanotubes de carbone sont des tubes composés d'atomes de carbone, et de diamètre de taille nanométrique, utilisés dans de nombreux secteurs industriels. Il existe une infinie variété de nanotubes de carbone: ils peuvent être souples ou rigides, à double ou à multiples parois, de longueur plus ou moins importante.. Autant de différences qui se traduisent sur les profils de risque. Il en résulte des difficultés pratiques pour savoir si un nanotube donné doit faire l'objet d'un dossier spécifique, ou peut être enregistré avec d'autres. C'est la question que se posent des industriels, par exemple, produisant un grand nombre de nanotubes pour des applications industrielles diverses, et dont les caractéristiques physico-chimiques sont variables ${ }^{9}$.

\section{Constituer de nouvelles catégories réglementaires}

L'approche « au cas par cas » repose donc sur un examen approfondi des critères permettant de différencier les objets « nano » de leurs équivalents « non nano », ainsi que les objets « nano » entre eux. Cet examen est mené dans le cadre des « Projets de mise en œuvre de REACH » (REACH Implementation Projects), qui visent à « s'assurer que toutes les parties prenantes, avant tout l'industrie et les autorités publiques, sont préparées de façon juin 2012. 
adéquate à l'application pratique de $\mathrm{REACH} »^{10}$. Les « parties prenantes » impliquées sont avant tout des fédérations des industries des nanotechnologies (Nanotechnology Industries Association) et de la chimie (European Chemicals Industry Council), des représentants de la Commission européenne et de l'ECHA, et, par l'intermédiaire d'un Stakeholder consultation group, des experts des États membres, des entreprises et d'ONG (autant d'acteurs également sollicités pour commenter les rapports intermédiaires et le rapport final). Les conclusions de ces projets, rendues en 2012, mettent en évidence la difficulté à repérer les critères de différenciation pertinents : " aucun consensus » n'a été atteint sur l'identification d'un ensemble d'équivalents nanométriques de substances chimiques connues (l'argent, le carbonate de calcium ou le dioxyde de titane) ${ }^{11}$.

Le détail des travaux entrepris lors de ces projets importe ici moins que la caractérisation du mode de gouvernement des substances par la précaution réglementaire. L’approche « au cas par cas » permet aux institutions européennes de réduire la question de l'enregistrement des nanomatériaux à un problème d'identification technique des substances sans avoir à constituer une nouvelle réglementation qui s'appliquerait aux activités industrielles indépendamment des particularités de chaque nanomatériau. Mais cette réduction entraîne nécessairement l'examen, potentiellement infini, des variations entre chaque nanomatériau.

\section{Des alternatives à la démarche « au cas par cas »}

Les initiatives de certains États membres permettent d'envisager des alternatives à l'approche au cas par cas. En 2012, la France introduit une définition légale des nanomatériaux, les « substances à l'état nanoparticulaire » afin d'imposer aux industriels une déclaration de ces substances auprès des autorités publiques nationales ${ }^{12}$. Cette initiative est une innovation réglementaire, qui crée une nouvelle catégorie - les «substances à l'état nanoparticulaire » - inconnue du droit, mais aussi des scientifiques. Elle est très critiquée : scientifiques et juristes lui reprochent la faible standardisation

10 <http://ec.europa.eu/environment/chemicals/reach/preparing/>, texte de présentation des REACH Implementation Projects, notre traduction

11 REACH Implementation Project on Nanomaterials, concluding remarks.

12 Décret $n^{\circ}$ 2012-232 du 17 février 2012 relatif à la déclaration annuelle des substances à l'état nanoparticulaire pris en application de l'article L. 523-4 du Code de l'environnement. 
des instruments permettant d'assurer la mesure des critères définissant ces « substances à l'état nanoparticulaire » (Lacour, 2012). La critique se rapproche de celle formulée par des toxicologues qui appellent à « ne pas définir les nanomatériaux » de peur de devoir fonder une nouvelle catégorie sur des décisions qui seraient alors « non scientifiquement valides » (Maynard, 2011). Mais pour les fonctionnaires impliqués, l'initiative est une façon (nouvelle) de traiter l'incertitude, qui introduit une catégorie ciblant les nanomatériaux « susceptibles d'émettre des nanoparticules potentiellement dangereuses » 13 (d'où l'expression « état nanoparticulaire »). Ces fonctionnaires savent que la stabilité technique n'est pas garantie, mais considèrent qu'elle permet « d'agir dans une situation où le gouvernement doit repérer ce que fait l'industrie ${ }^{14}$.

À la suite de positionnements explicites de la France en faveur de dispositions spécifiques aux nanomatériaux dans $\mathrm{REACH}^{15}$, l'initiative française des « substances à l'état nanoparticulaire » est conçue comme une démonstration auprès des institutions européennes de la possibilité d'introduire des mesures spécifiques aux nanotechnologies dans REACH, ce qu'un fonctionnaire du ministère français de l'Écologie désigne par un « nano-patch » ${ }^{16}$. Ce « nano-patch » irait à l'encontre de l'approche « cas par cas » promue par la Commission dans la mesure où il introduirait une définition transversale des substances « nano ».

La Commission s’oppose également sur ce point au Parlement européen. En réponse à l'affirmation par la Commission de la validité de la réglementation existante pour la régulation des nanomatériaux, le Parlement exprime son désaccord:

« (The Parliament) does not agree [...] with the Commission's conclusions that a current legislation covers in principle the relevant risks relating to nanomaterials, and b) that the protection of health, safety and the environment needs mostly to be enhanced by improving implementation of current legislation ${ }^{17}$. »

Extrait d'entretien, chef de bureau des substances chimiques, février 2013.

14 Ibid.

15 Malgré la diversité des acteurs administratifs nationaux, la position française au sujet de l'application de REACH aux nanomatériaux a été constante, et toujours en faveur de la prise en compte de ces objets comme nouvelles substances (entretien, chef de bureau, direction générale de la Santé, octobre 2010).

16 Entretien, chef de bureau substances chimiques, février 2013.

17 EP resolution: 9 
C'est en suivant cette ligne que le Parlement entreprend une série d'initiatives législatives visant à faire exister les nanomatériaux comme catégorie dans la réglementation européenne, en agissant dans les domaines non traités par $\mathrm{REACH}$, notamment les produits biocides ou les aliments nouveaux ${ }^{18}$, ou les cosmétiques, qui font l'objet d'une réglementation spécifique indépendante de $\mathrm{REACH}^{19}$. Au cours de la révision des directives réglementant les cosmétiques, les aliments nouveaux et les biocides, le Parlement ajoute, à de larges majorités, des amendements qui requièrent des dispositions spécifiques pour les nanomatériaux, définis comme catégorie spécifique. En réponse à un amendement ajouté par le Parlement au règlement " cosmétiques » par exemple, les industriels sont tenus de faire figurer la mention « nano » dans la liste des ingrédients des produits contenant des nanomatériaux ${ }^{20}$. L’amendement les définit de la façon suivante:

«"nanomaterial" means an insoluble or biopersistent and intentionally manufactured material with one or more external dimensions, or an internal structure, on the scale from 1 to $100 \mathrm{~nm}^{21}$. »

Cette catégorie ambitionne de saisir les substances dont le caractère « nano » s'accompagne de risques à réglementer. C'est le sens des qualificatifs « insoluble » et «biopersistant ${ }^{22}$. Mais cette définition est aussi en partie arbitraire. La limite de taille (« entre 1 et $100 \mathrm{~nm} »$ ) est reprise des programmes nationaux de politique scientifique, qui l'utilisent comme une façon commode de regrouper un ensemble d'activités technologiques modifiant la matière à de petites échelles (Laurent, 2010). Elle permet de cibler un ensemble de substances de faible taille et comportant potentiellement de nouvelles propriétés, mais n'est pas un seuil en deçà duquel de nouveaux risques apparaîtraient systématiquement.

20 Regulation (EC) No 1223/2009 of the European Parliament and of the Council of 30 November 2009 on cosmetic products.

21 Ibid: Art. 2.1, alinea k.

22 Dans le règlement «Nouveaux Aliments » et la directive Biocides, les définitions introduites par le Parlement sont plus complexes, et s'appuient sur des critères comprenant, entre autres, la surface spécifique et la distribution de tailles. La Commission introduit en novembre 2011 une définition des nanomatériaux par le biais d'une recommandation sans valeur juridique contraignante, qui utilise des critères similaires. 
De même que celui de l'initiative française, le devenir des catégories créées par le Parlement est incertain. Les infrastructures techniques pour assurer la robustesse des mesures manquent et les possibilités de contrôle ne sont pas garanties. Mais là encore, l'opposition aux initiatives de la Commission permet de caractériser par contraste les ressorts de la précaution réglementaire. Le cas par cas tel qu'appliqué par la Commission se caractérise par la réticence à la création de nouvelles catégories qui ne soient appuyées sur une infrastructure technique et sociale robuste - au risque de repousser indéfiniment toute initiative légale contraignante. L'alternative, en l'occurrence, consiste alors à utiliser le droit comme outil permettant de créer des catégories techniques, si besoin arbitraires, pour les imposer aux acteurs industriels.

\section{Autoriser les phtalates}

\section{L'autorisation comme procédure globalisante}

Les phtalates sont présents dans une multiplicité de secteurs, de la construction aux dispositifs médicaux, mais aussi dans de très nombreux produits de consommation contenant des matières plastiques. En juin 2008, trois dossiers correspondant aux trois phtalates les plus utilisés (DEHP, DBP et BBP) sont proposés par deux États membres, la Suède et l'Autriche, en vue d'une identification comme substances « extrêmement préoccupantes ». Cette initiative est une première étape vers l'inscription de ces substances à l'Annexe XIV de REACH, qui regroupe les substances qui ne pourront plus être mises sur le marché sans autorisation après une date définie par les autorités européennes. Après sélection par l'ECHA et validation par la Commission européenne, les substances concernées sont interdites, sauf autorisation exceptionnelle (d'où le nom de procédure d'autorisation) pour un industriel et un usage donné. Il s'agit donc d'une approche inclusive qui interdit a priori tous les usages de ces substances. Après avoir été désignés comme prioritaires en raison de leur classification comme substances toxiques pour la reproduction, la décision d'inclure les trois phtalates à l'annexe XIV est publiée en 2011 pour une interdiction effective début 2015.

Les phtalates font partie des premières substances traitées par la procédure d'autorisation. Les mesures réglementaires appliquées aux phtalates semblent au premier abord consensuelles pour les États membres, la Commission et l'ECHA, qui s’appuient sur des études concordantes démontrant la dangerosité 
des phtalates (Commission européenne, 2008). Définir les phtalates de manière globale mettait alors en évidence la capacité de la procédure d'autorisation à interdire a priori tous leurs usages. Pour les experts des agences sanitaires responsables de ces dossiers, les phtalates étaient un «beau » cas pour pratiquer la procédure d'autorisation ${ }^{23}$. C'est ce que suggèrent en entretiens les industriels, mais aussi les représentants des autorités françaises. Pour une responsable « gestion des produits REACH » de l'Union des industries chimiques par exemple, le choix de substances bien connues a permis aux autorités européennes d'introduire une « vraie dimension de légitimation » ${ }^{24}$ de la procédure. Le cas des phtalates permet de montrer que la procédure fonctionne en proposant une démonstration de sa capacité à interdire.

Les nanomatériaux ont fourni une illustration des difficultés que peut rencontrer la procédure d'enregistrement, et en particulier de l'approche au cas par cas, réticente à constituer des catégories réglementaires globales. L'autorisation des phtalates semble présenter un fort contraste: une catégorie ciblant un ensemble de substances vouées à être retirées du marché européen est constituée par la procédure d'autorisation. Des initiatives prises par certains États membres incitent néanmoins à nuancer cette première lecture. Considérons par exemple une proposition de loi du Parlement française, transmise au Sénat en 2011, visant à « interdire l'utilisation des phtalates, des parabènes et des alkylphénols » (Assemblée nationale, 2010). L'examen du projet de loi en commission permet aux parlementaires de revenir sur les motivations de cette initiative:

« REACH est moins ambitieu[x] que ce qui était prévu au départ. [...] De grâce, ne recommençons pas les mêmes erreurs qu'avec l'amiante ou avec les éthers de glycol! Dès lors que l'on ne maîtrise pas les effets sanitaires de ces molécules, nous devons appliquer le principe de précaution ${ }^{25}$. »

«Appliquer le principe de précaution », ici, consiste à interdire les phtalates grâce à la contrainte réglementaire. Il s'agirait d'être «plus ambitieux » que REACH. En quoi la procédure d'autorisation des phtalates est-elle donc « moins ambitieuse » qu'une interdiction? Pour le comprendre, et donc pour comprendre les ressorts de l'initiative française, il est nécessaire d'examiner en détail la construction des catégories réglementaires par la procédure d'autorisation.

23 Entretien avec un membre du comité d'analyse socio-économique de l'ECHA, octobre 2012

24 Entretien avec une responsable gestion des produits REACH à I'Union des Industries Chimiques, octobre 2012. 


\section{Exclure les articles importés et les substances exportées}

L'autorisation des phtalates s'appuie sur un découpage permettant de sélectionner les objets sur lesquels la réglementation a prise. Une première exception à l'application de l'autorisation concerne d'abord les articles importés. REACH définit les « articles » comme des « objets auxquels sont donnés, au cours du processus de fabrication, une forme, une surface ou un dessin particuliers qui sont plus déterminants pour sa fonction que sa composition chimique ». Ce sont en d'autres termes des produits manufacturés. Or la procédure d'autorisation ne s'applique pas aux articles importés, même si ceux-ci contiennent des substances interdites : « les substances constituant une partie (intégrante) d'articles importés ne peuvent pas faire l'objet d'une autorisation. Cela signifie qu'afin d'importer des articles à l'intérieur de l'EEE, une autorisation ne peut pas être exigée » (European Chemicals Agency, 2011). Ce cas est particulièrement répandu pour les phtalates :

«Les phtalates sont présents dans tous les articles en plastique, de la boîte à la toile cirée en passant par le dispositif médical : on retrouve des phtalates dans les articles qui arrivent sur le marché communautaire. [...] REACH a été vu à un moment comme un instrument de protectionnisme, mais ce n'est pas aussi simple que ça ${ }^{26}$. »

Dans cet extrait, une fonctionnaire française responsable de REACH suggère que l'exclusion des articles importés dans la procédure d'autorisation est à rapprocher des discussions menées lors de l'écriture du règlement REACH. Pendant les négociations du règlement, les États-Unis ont en effet accusé REACH d'être défavorable aux importations, jusqu’à le qualifier de protectionniste:

"Quand REACH a commencé à être discuté de façon approfondie, certains opposants les plus virulents à REACH ont été les Américains. Des délégations de l'administration américaine et des lobbyistes ont essayé d'expliquer à la Commission européenne que c'était absolument scandaleux, que tout ça n'était qu'une mesure de protectionnisme déguisé, et que c'était totalement inacceptable ${ }^{27}$. » chimiques du Ministère de l'Ecologie, septembre 2012.

27 Entretien avec un ancien responsable du Conseil européen de l'industrie chimique (CEFIC), novembre 2012 
L'exclusion des articles importés du champ d'application de l'autorisation est alors pour certains acteurs des négociations de REACH un moyen d'éviter que l'outil réglementaire ne soit jugé protectionniste. Elle a pour conséquence le fait que les importateurs européens d'articles produits hors Union ne sont pas inquiétés par l'inclusion des phtalates dans la liste XIV.

Une seconde exception à l'application de l'autorisation concerne les industriels européens qui exportent leur production de substances. Les producteurs européens de substances exportées en dehors de l'Union bénéficient du fait que la procédure d'autorisation requiert l'identification d'un usage par l'entreprise productrice. Si l'intégralité de la production d'une substance est exportée, aucun usage n’en est fait sur le marché européen. En conséquence, un producteur de phtalates exportant sa production en dehors de l'Union n'est pas concerné par la procédure d'autorisation. En fin ce compte, le découpage de la catégorie réglementaire « phtalates » au sens de la procédure d'autorisation élimine du champ des restrictions un ensemble d'objets. Cette mesure illustre une volonté de contrôler le marché des substances chimiques dangereuses tout en prenant en compte un ensemble de contraintes liées au commerce international. Le régulateur européen fait preuve de précaution au sens où il cherche à contrôler des objets risqués, mais aussi lorsqu'il manipule la contrainte réglementaire. Au contraire de cette démarche de précaution réglementaire, l'initiative française mentionnée plus haut consiste à éviter les découpages à l'intérieur de l'ensemble des phtalates pour cibler une catégorie générale devant faire l'objet d'une interdiction.

\section{Reconstituer une approche au cas par cas}

Le découpage de la catégorie des phtalates au sein de la procédure d'autorisation n'exclut pas seulement de son champ d'application les articles importés et les substances exportées. Un ensemble d'exemptions est accordé en fonction des usages des phtalates, que les interventions du Parlement européen permettent de mettre en lumière. Le Parlement s'est par exemple inquiété, en 2011, du flou relatif à la réglementation de l'utilisation des phtalates dans les jouets ${ }^{28}$. Des parlementaires ont jugé problématique le fait que des possibilités d'exception au cas par cas existent, dans un domaine (les jouets) qui faisait avant l'introduction de REACH l'objet de restrictions spécifiques. 
Face au Parlement, la Commission affirme, en réponse aux questions soulevées par ces parlementaires, qu'une « interdiction totale sans aucune possibilité d'exemption ne serait pas justifiable ni réaliste », et que l'évaluation des phtalates doit se faire «au cas par cas, sur la base de l'information scientifique disponible pour chaque substance ${ }^{29}$. On retrouve ce processus par le biais de dispositions prévues par le texte, qui amendent indirectement la procédure d'autorisation. REACH permet de créer des exceptions aux interdictions qui seraient décidées dans le cadre de son application:

«Des utilisations ou catégories d'usages peuvent être exemptées de l'obligation d'autorisation, à condition que, compte tenu de la législation communautaire spécifique existante, [...] le risque soit bien maîtrisé ${ }^{30}$. »

À rebours de la catégorie générale constituée par la procédure d'autorisation, cette clause du texte permet finalement de créer des catégories distinctes en fonction des usages. Ce retour de l'approche au cas par cas a une justification scientifique, car il permet de préciser l'évaluation du risque. Les expositions aux phtalates en effet ne sont pas les mêmes suivant les usages. Les rapports d'évaluation du risque des phtalates s'intéressent ainsi à des scénarios d'exposition différents pour des travailleurs, des consommateurs ou de personnes exposées via l'environnement (Commission européenne, 2008), qui se traduisent par des niveaux de risque différents.

Un peu à la manière des « REACH Implementation Projects » mentionnées dans le cas des nanomatériaux, les réunions du CARACAL ${ }^{31}$, un groupe d'experts composé de représentants des autorités compétentes des États membres qui conseillent la Commission et l'ECHA, sont aussi l'occasion pour ses participants ${ }^{32}$ de débattre de l'identification d'usages distincts, qui supposeraient des aménagements différenciés des contraintes réglementaires. C'est ainsi que le CARACAL a confirmé l'existence de possibilités d'exemptions pour peu que les usages visés soient déjà couverts par une législation communautaire existante (Commission européenne, 2010). Cette démarche a permis aux firmes pharmaceutiques d'exclure les « substances

Réponse du 2 juin 2011 donnée par M. Tajani au nom de la Commission à la question de Frédérique Ries.

30 Article 58 \$2 du règlement $\mathrm{REACH}$

31 Acronyme anglais pour Competent Authorities for REACH and CLP. En France, il s'agit du Ministère de l'Ecologie.

32 Début 2014, le CARACAL accueillait une trentaine de parties prenantes comme « observateurs »: organisations internationales, associations professionnelles, ONG, ou encore des pays en dehors de I'UE. 
qui composent l'emballage de médicaments » du champ de l'autorisation. En montrant que cet usage était réglementé par ailleurs, dans la mesure où une demande d'autorisation de mise sur le marché de médicaments «peut inclure » la composition de leurs emballages, les industriels ont identifié une nouvelle catégorie (celle des emballages de substances actives) qui permet de limiter les contraintes introduites par $\mathrm{REACH}^{33}$.

Cette dernière manœuvre complète le tableau du fonctionnement de la procédure d'autorisation avec les phtalates. Les découpages réglementaires (entre « substances » et « articles », entre différents usages) permettent de répondre à la critique du protectionnisme, et à celle de la surabondance des normes, mais aussi d'examiner plus en détail les risques des substances en fonction des situations particulières d'utilisation. Il s'agit ici de faire avec un ensemble de contraintes : celles du commerce international, de la cohérence entre les différents textes réglementaires, mais aussi les contraintes techniques de l'évaluation du risque. De même que le cas par cas permet dans le cas des nanomatériaux une description scientifiquement plus fine des risques des objets, de même la distinction entre les usages des phtalates permet de développer une évaluation attentive aux différents scénarios d'exposition. Comme pour les nanomatériaux, le corolaire de cette exploration fine « au cas par cas » réalisant l'approche de précaution réglementaire adoptée par la Commission est la réticence à introduire des contraintes légales fortes pour poursuivre toujours l'exploration technique. À l'exploration des contraintes économiques et techniques dans un ensemble de lieux de négociations répondent des initiatives des États membres ou du Parlement constituant des catégories plus générales, insensibles à la variété des « cas » possibles, et associées à des contraintes juridiques plus fortes adressées aux industriels. sur le marché de médicaments incluent la composition de leurs emballages. La réalité est un peu différente, dans la mesure où les textes qui mentionnent cette possibilité sont de simples lignes directrices non contraignantes. Cf. EMEA 2005, Guideline on plastic immediate packaging materials, EMEA/ CVMP/205/04. 


\section{Gouverner par la précaution réglementaire}

\section{La précaution réglementaire comme mode d'action publique}

Les cas des nanomatériaux et des phtalates permettent d'illustrer le mode de fonctionnement des deux principaux volets de $\mathrm{REACH}$, la procédure d'enregistrement et la procédure d'autorisation. Dans le cas des nanomatériaux, les incertitudes techniques sont maximales et l'application préconisée par la Commission de la procédure d'enregistrement consiste à adopter une démarche au cas par cas qui ne bouleversera pas l'appareil législatif existant. Les phtalates semblent fournir l'illustration d'un cas de certitude scientifique permettant de faire fonctionner la procédure d'autorisation, plus restrictive. Pourtant, le découpage de la catégorie des phtalates permet aux substances exportées et aux articles importés d'échapper à la procédure, tandis qu'une approche au cas par cas est à nouveau entreprise alors que les multiples usages des phtalates sont examinés.

Ces deux exemples permettent de repérer une façon de conduire l'action publique européenne caractérisée par la précaution réglementaire. La notion de précaution réglementaire permet de saisir une façon d'agir de l'administration européenne qui cherche à faire avec les exigences relatives au principe de précaution et les réticences, dans le cadre de l'objectif de " meilleure réglementation », à étendre les contraintes réglementaires. Dans les deux cas examinés ici, la précaution réglementaire décrit un usage de la réglementation faisant des substances chimiques des objets de gouvernement grâce à la multiplication de « cas », mais aussi une grande mesure dans la manipulation de la contrainte réglementaire.

L'analyse de REACH et de sa mise en œuvre dans les termes de la précaution réglementaire permet ainsi de reprendre à nouveau frais la question de la légitimité de l'action publique européenne. Les mécanismes sur lesquels REACH s'appuie peuvent être décrits dans les termes utilisés par les politistes et juristes ayant cherché à catégoriser les formes de la légitimité démocratique européenne, notamment dans le recours à l'expertise au niveau européen (Robert, 2010). On a vu dans les descriptions précédentes que le système de procédures encadrant les décisions entre la Commission et les États membres, la « comitologie », est utilisé dans la mise en œuvre du règlement, notamment l'inscription des substances à l'Annexe XIV en vue de leur autorisation. À ces dispositions s'ajoutent des procédures ad hoc, au sein d'espaces aussi bien «techniques » que «politiques » et dont les 
logiques de composition pourraient faire l'objet d'un travail à part entière (Fouilleux et al., 2005 ; Gornitzka et Sverdrup, 2010 ; Robert, 2003). Les « Reach Implementation Projects », dont on a vu l'intervention dans le cas des nanomatériaux, sont menés par des groupes d'experts associant fonctionnaires de la Commission et de l'ECHA, représentants des États membres et des industriels. D'autres espaces réunissent plus ou moins formellement ces mêmes acteurs. Le CARACAL, rencontré dans le cas des phtalates lors $\mathrm{du}$ retour à une approche au cas par cas, organise des sessions ouvertes auxquelles participent de nombreux observateurs, notamment industriels, tandis que les sessions fermées ne réunissent que l'ECHA, la Commission et les États membres.

Certains commentateurs qualifient ainsi REACH « d'objet hybride », qui n'est réductible ni à l'action contraignante de la réglementation ni aux processus d'intégration volontaires fondés notamment sur la Méthode Ouverte de Coordination (Hey et al., 2007). En conséquence, la légitimité de cet « objet hybride » ne peut être réductible à celle de « l'état régulateur » dont parle Majone pour qualifier l'action publique européenne (Majone, 1996) ni à l'exercice de la délibération dans des espaces comme ceux prévus par la comitologie (Joerges et Neyer, 1997). La division du travail entre des acteurs aussi multiples que l'ECHA, les agences d'expertise nationales, les Ministères des États membres, les industriels sous forme ou non de coalitions, les ONG, la Commission, les parlements nationaux et européen, implique une distribution des espaces de discussion de la mise en œuvre de REACH : Parlements, Commission, consultations publiques de l'ECHA, comités d'experts, etc., et par le biais de diverses opérations (comitologie, délégation au règlement, intervention des agences d'expertise...).

À travers les instruments de la précaution réglementaire, la légitimité de l'action publique européenne apparaît alors comme le résultat d'un ensemble de négociations distribuées dans des arènes multiples. Ceci se traduit de deux façons. D'une part, en offrant la possibilité d'un retraçage des catégories, y compris dans les procédures les plus restrictives. D’autre part, en déployant la négociation entre diverses parties prenantes jusque dans les détails techniques les plus fins, afin d'explorer les caractéristiques techniques des substances chimiques ou les modalités de leurs usages dans le cadre d'approches au cas par cas. Au final, la précaution réglementaire aboutit à la réalisation d'actions mesurées, visant à cibler au maximum les lieux d'intervention réglementaire. 


\section{Un ordre constitutionnel caractérisé par la précaution réglementaire}

Les processus fondés sur la précaution réglementaire sont loin d'être neutres. Premièrement, ils conduisent à répartir les pouvoirs au sein des institutions européennes. Ils donnent un rôle central à la Commission et aux agences dans le fonctionnement réglementaire, en distinguant le règlement (le texte législatif discuté par l'ensemble des institutions européennes) et sa mise en application. En parallèle, la précaution réglementaire répartit la discussion sur la mise en application de REACH dans une multitude de lieux à faible visibilité. Elle donne la possibilité aux États membres d'intervenir dans différents sites (comme la demande d'inclusion à l'annexe XIV, ou la participation aux REACH Implementation Projects) au cours des procédures prévues par REACH. Elle permet à des experts nationaux, européens et industriels d'échanger autour des modalités de la définition des substances (comme les nanomatériaux) ou de leurs usages (comme ceux des phtalates). Elle rend donc l'exercice de la subsidiarité plus complexe qu'une simple délégation aux instances européennes ou aux États.

Deuxièmement, l'accès aux discussions qu'implique la précaution réglementaire est inégal. La précaution réglementaire entraîne nécessairement des effets d'asymétrie, du fait des ressources nécessaires pour intervenir dans la multiplicité des lieux où se discute l'approche au cas par cas. Pour participer aux négociations, il est en effet nécessaire d'investir un grand nombre de groupes de travail, mais aussi de disposer des connaissances techniques permettant d'examiner en détail un grand nombre de cas particuliers. L'intervention des fédérations d'associations environnementales est significative à cet égard. Par exemple, le Bureau européen de l'environnement s'est investi dans la négociation ayant conduit à une « recommandation » de la Commission (sans valeur juridique contraignante) quant à la définition du terme « nanomatériaux », mais n'a pu participer à l'ensemble des initiatives menées dans le cadre des REACH Implementation Projects sur les nanomatériaux (dont les impacts juridiques sont bien plus significatifs) ${ }^{34}$.

Enfin, la précaution réglementation conduit à étendre les explorations techniques et sociales, au risque de multiplier les cas insolubles. Comment en effet prétendre être exhaustifs sur les usages des phtalates ou sur l'infi- 
nie variation des caractéristiques physico-chimiques des nanomatériaux? Comment s'assurer que toutes les parties prenantes ont été entendues? La précaution réglementaire conduit nécessairement à ne prendre des décisions contraignantes qu'avec le plus grand soin, au risque de repousser toujours le moment de trancher pour introduire des raffinements supplémentaires dans l'approche au cas par cas.

La sociologue américaine Sheila Jasanoff propose d'utiliser la notion de « constitution » en étendant son sens juridique initial pour englober à la fois la définition et la répartition des pouvoirs au sein des institutions politiques et la constitution d'objets de gouvernement. Comprise ainsi, la notion de constitution permet de saisir les processus à la fois normatifs et ontologiques de mise en ordre de la réalité (Jasanoff, 2011a). À la suite de Jasanoff, on peut alors parler « d'ordre constitutionnel » pour désigner un type d'organisation articulant construction des institutions réglementaires et d'expertise, des sujets politiques, et des objets à gouverner (Jasanoff, 2011b; Laurent, 2013). La précaution réglementaire apparaît ainsi comme la caractéristique d'un ordre constitutionnel européen défini par l'action mesurée, la multiplication des lieux de l'expertise collective (mais non accessible à tous), et la production de multiples catégories réglementaires. Que cet ordre constitutionnel articule étroitement des considérations ontologiques (sur la nature des entités traitées) avec des positions normatives (sur ce qu'il est désirable de poursuivre au niveau européen) est très visible lorsqu'on se penche sur les oppositions adressées à la Commission européenne.

Dans les deux sections précédentes, on a vu en effet que des États membres et le Parlement européen considèrent que l'approche de la Commission n'est pas satisfaisante. Le ressort de ces critiques est précisément d'attaquer le faible pouvoir contraignant de la précaution réglementaire, ou, du moins, le risque de reporter toujours à plus tard la décision en situation d'incertitude. Les alternatives proposées sont fondées sur la construction de nouvelles catégories réglementaires indépendantes des particularités des « cas »: des « nanomatériaux » introduits dans des directives comme la directive « cosmétiques » et définis par des critères faiblement outillés techniquement, des phtalates définis indépendamment de leurs usages. Plutôt que l'action mesurée, ces alternatives imposent la contrainte légale. Plutôt que l'examen technique minutieux, elles assument l'intervention tranchée, définissant des catégories alors que l'infrastructure technique permettant de les identifier n'est pas toujours en place. Elles proposent d'introduire de nouvelles catégories réglementaires en situation d'incertitude scientifique. Elles suggèrent des 
articulations différentes entre les interventions ontologiques et normatives. De ce fait, ces initiatives problématisent la légitimité de l'action publique européenne d'une façon différente de la précaution réglementaire. Elles cherchent à rendre possibles les interventions des États membres et du Parlement face à la gestion au cas par cas par la Commission et ses agences.

\section{Conclusion}

La référence à la «précaution » dans les politiques publiques européennes fait l'objet de deux critiques. L'une déplore que l'usage du terme ne donne pas lieu à des réglementations plus contraignantes des activités industrielles. L'autre fait de la « précaution » le signe d’une spécificité des pays européens, devenus réticents à accepter l'innovation technique et les risques inévitables qui l'accompagnent. Dans cet article, nous nous sommes distanciés de ces deux critiques, en cherchant à caractériser les formes de l'action publique européennes sur les objets techniques. Il s'agissait de ne pas prendre la « précaution » comme une catégorie donnée, mais d'explorer sa traduction dans des instruments de gouvernement. La construction de catégories réglementaires pour les objets techniques s'est révélée être un site empirique particulièrement riche à cet égard. En analysant les procédures d'enregistrement et d'autorisation du règlement $\mathrm{REACH}$, nous avons ainsi vu en acte un mode de gouvernement caractérisé par la «précaution réglementaire ». En utilisant le règlement dans un objectif de précaution tout en étant précautionneux dans la manipulation de la contrainte réglementaire, les institutions européennes associent la production de multiples catégories de description des substances au fil d'approches « au cas par cas » et la distribution de la discussion collective dans de multiples espaces. Elle conduit à faire des incertitudes un problème technique d'exploration collective potentiellement sans fin. L'intérêt de l'approche adoptée ici, outre qu'elle permet de donner un contenu empirique à l'affirmation de l'objectif de la « précaution », suggère une voie pour les études de l'intégration européenne qui permet d'analyser ses dimensions constitutionnelles au sens le plus fort du terme. En se penchant sur les processus qui articulent étroitement constructions ontologiques (quelles sont les entités gouvernables?) et considérations normatives (qu'est-ce qui est souhaitable pour le futur européen?), l'analyse permet de montrer l'importance des questions soulevées par l'action réglementaire sur les objets techniques. 


\section{Références bibliographiques}

Assemblée nationale (2010), Proposition de loi visant à interdire l'utilisation des phtalates, des parabènes et des alkylphénols, Paris.

Bowker Geoffrey and Star Susan Leigh (1999), Sorting things out. Classification and its consequences, Cambridge, MIT Press.

Commission européenne (2000), Communication from the Commission on the Precautionary Principle, Bruxelles, Office for Official Publications of the European Communities.

Commission européenne (2008), EU Risk Assessment Report on DEHP.

Commission européenne (2010), 5th meeting of Competent Authorities for REACH and CLP, 15-17 June 2010.

Dratwa Jim (2002), « Taking risks with the precautionary principle: food (and the environment) for thought at the European Commission ", Journal of Environmental Policy and Planning, vol. 4, $\mathrm{n}^{\circ}$ 3, p. 197-213.

Dupuy Jean-Pierre (2003), « Principe de précaution et catastrophisme éclairé », Les Cahiers du M.U.R.S., $\mathrm{n}^{\circ} 42$, p. 6-25.

European Chemicals Agency (2011), Guide des exigences applicables aux substances contenues dans des articles, Helsinki.

Fisher Elizabeth (2008), « The "Perfect Storm” of Reach: Charting Regulatory Controversy In The Age Of Information, Sustainable Development, and Globalization ", Journal of Risk Research, vol. 11, n 4, p. 541-563.

Fouilleux Eve, de Maillard Jacques and Smith Andy (2005), « Technical or Political? The Working Groups of the EU Council of Ministers », Journal of European Public Policy, vol. 12, n 4, p. 609-623.

Fuchs Olivier (2009), « REACH: A New Paradigm for the Management of Chemical Risks », Health and Environment Reports, n 4, p. 1-113.

Godard Olivier (1999), «De l'usage du principe de précaution en univers controversé: entre débats publics et expertise », Futuribles, n 238-240, p. 37-60.

Godard Olivier (2003), « Le principe de précaution comme norme de l'action publique, ou la proportionnalité en question ", Revue économique, vol. 54, $\mathrm{n}^{\circ}$ 6, p. 1245-1276.

Gornitzka Åse et Sverdrup Ulf (2010), « Enlightened decision making? », Politique européenne, $\mathrm{n}^{\circ} 32$, p. 125-149. 
Hey Christian, Jacob Klaus and Volkery Axel (2007), « Better Regulation by New Governance Hybrids? Governance Models and The Reform of European Chemicals Policy », Journal of cleaner production, $\mathrm{n}^{\circ}$ 15, p. 1859-1874.

Jasanoff Sheila (2004), States of Knowledge. The Coproduction of Science and Social Order, London, Routledge.

Jasanoff Sheila (2011a), « Constitutional Moments in Governing Science and technology », Science and engineering ethics, vol. 17, n 4, p. 621-638.

Jasanoff Sheila (2011b), Reframing Rights: Bioconstitutionalism in the Genetic Age, Cambridge, MIT Press.

Joerges Christian and Neyer Jürgen (1997), « From Intergovernmental Bargaining To Deliberative Political Processes : The Constitutionalisation Of Comitology », European law journal, vol. 3, n 3 , p. 273-299.

Jouzel Jean-Noël et Lascoumes Pierre (2011), « Le règlement REACH : une politique européenne de l'incertain. Un détour de régulation pour la gestion des risques chimiques ». Politique européenne, $\mathrm{n}^{\circ} 33$, p. 185-214.

Lacour Stéphanie (2012), « L'impossible définition des substances à l'état nanoparticulaire », Revue environnement et développement durable, $\mathrm{n}^{\circ} 5$, étude 8.

Larrère Catherine (2003), "Le principe de précaution et ses critiques », Innovations, vol. 18, $\mathrm{n}^{\circ} 2$, p. 9-26.

Lascoumes Pierre (1996), « La précaution comme anticipation des risques résiduels et hybridation de la responsabilité », L’Année sociologique, vol. 46, $\mathrm{n}^{\circ} 2$, p. 359-382.

Laurent Brice (2010), Les politiques des nanotechnologies, Paris, Charles Léopold Mayer.

Laurent Brice (2013), « Du laboratoire scientifique à l'ordre constitutionnel », Raisons politiques, 2013/2, n ${ }^{\circ}$ 50, p. 137-155.

Levidow Les (2001), " Precautionary Uncertainty: Regulating GM Crops in Europe », Social Studies of Science, vol. 31, n 6 , p. 842-874.

Lezaun Javier (2006), « Creating a New Object of Government Making Genetically Modified Organisms Traceable », Social Studies of Science, vol. $36, \mathrm{n}^{\circ} 4$, p. 499-531.

Loubière Sandrine, Rotily Michel, Durand-Zaleski Isabelle, Costagliola Dominique et Moatti Jean-Paul (2001), «L'introduction de la PCR dans le dépistage du virus de l'hépatite $\mathrm{C}$ dans les dons de sang: du mésusage du principe de précaution », Médecine/sciences, vol. 17, nº 3, p. 345-349. 
Majone Giandomenico (1996), La Communauté européenne: un Etat régulateur, Paris, Montchrestien.

Marchant Gary Elvin and Mossman Kenneth L. (2004), Arbitrary and Capricious: The Precautionary Principle in the European Union courts, Washington, D.C, AEI Press.

Maynard Andrew D. (2011), « Don’t Define Nanomaterials », Nature, vol. 475, p. 31.

Robert Cécile (2003), « L'expertise comme mode d'administration communautaire. Entre logiques technocratiques et stratégies d'alliance », Politique européenne, $\mathrm{n}^{\circ} 11$, p. 57-78.

Robert Cécile (2010), «Les groupes d'experts dans le gouvernement de l'Union européenne. Bilans et perspectives de recherche », Politique européenne, $\mathrm{n}^{\mathrm{o}} 32, \mathrm{p} .7-38$.

de Sadeleer Nicolas (2001), « Le statut juridique du principe de précaution en droit communautaire: du slogan à la règle », Cahiers de droit européen, vol. $37, \mathrm{n}^{\circ} 1-2$, p. 91-132.

Stokes Elen (2008), « The EC Courts' Contribution to Refining the Parameters of Precaution », Journal of Risk Research, vol. 11, n 4, p. 491-507.

Tosun Jale (2013), « How the EU Handles Uncertain Risks: Understanding the Role of The Precautionary Principle », Journal of European Public Policy, vol. 20, $\mathrm{n}^{\circ} 10$, p. 1517-1528.

Vogel David (2012), The Politics of Precaution: Regulating Health, Safety, and Environmental Risks in Europe and the United States, Princeton, Princeton University Press.

\section{Henri Boullier}

Doctorant en sociologie à l'Université Paris-Est et rattaché au Laboratoire interdisciplinaire Sciences, Innovations, Sociétés (LISIS), et à l'Institut francilien recherche Innovation société (IFRIS).

henri.boullier@enpc.fr

\section{Brice Laurent}

Chargé de recherche au Centre de sociologie de l'innovation (CSI) de Mines ParisTech

brice.laurent@mines-paristech.fr. 